\title{
New approaches in determining the mass of the top quark: alternative techniques and differential measurements
}

Stijn Blyweert ${ }^{* \dagger}$

Vrije Universiteit Brussel

E-mail: stijn.blyweertacern.ch

\begin{abstract}
Measurements of the top-quark mass employing alternative methods are presented using data collected by the CMS experiment in proton-proton collisions at the LHC in the years 2011 and 2012 at centre-of-mass energies of 7 and $8 \mathrm{TeV}$. The alternative methods include the use of endpoint distributions and the use of the lifetime of the b-hadron, as well as the study of possible model dependencies of the top-quark mass measurement on the event kinematics. Measurements of the difference between the masses of top and antitop quarks are also presented. Furthermore, the top-quark mass and also $\alpha_{s}$ are extracted from the measured top-quark pair cross section.
\end{abstract}

The European Physical Society Conference on High Energy Physics -EPS-HEP2013

18-24 July 2013

Stockholm, Sweden

\footnotetext{
*Speaker.

†n behalf of the CMS Collaboration.
} 


\section{Introduction}

The top quark, which was discovered in 1995 at the Tevatron, is the heaviest currently known fundamental particle. Its mass is an important input parameter of the global electroweak fits. Since the mass of the Brout-Englert-Higgs boson is now known, the electroweak fits can be used to perform consistency checks of the Standard Model.

The top-quark mass $m_{t}$ has been measured by the Tevatron and LHC experiments with great precision. A further improvement of the precision of these measurements and of the understanding of what these measurements actually mean is necessary. Therefore a number of alternative topquark mass measurements are performed with the data collected by the CMS experiment [1] in 2011 and 2012.

\section{Kinematic dependence of the measured top-quark mass}

In the first study presented here the top-quark mass was measured in bins of several kinematic observables to confirm the good modelling of the top-quark mass observable by simulation and to check the sensitivity of the top-quark mass observable to different effects like for example colour reconnection or the underlying event. This study was based on the entire 2011 dataset and uses the Ideogram method to perform a two-dimensional measurement of the top-quark mass and the jet energy scale [2] which is currently the most precise top-quark mass measurement from CMS.

Exactly the same analysis is repeated and either the top-quark mass $m_{t}$ and the jet energy scale JES measured with the two-dimensional technique ( $m_{t}$ and JES), or the top-quark mass measured with the one-dimensional technique is plotted in bins of 12 kinematic variables [3]. Some examples of these binned measurements are shown in Figure 1. In general a good agreement between the
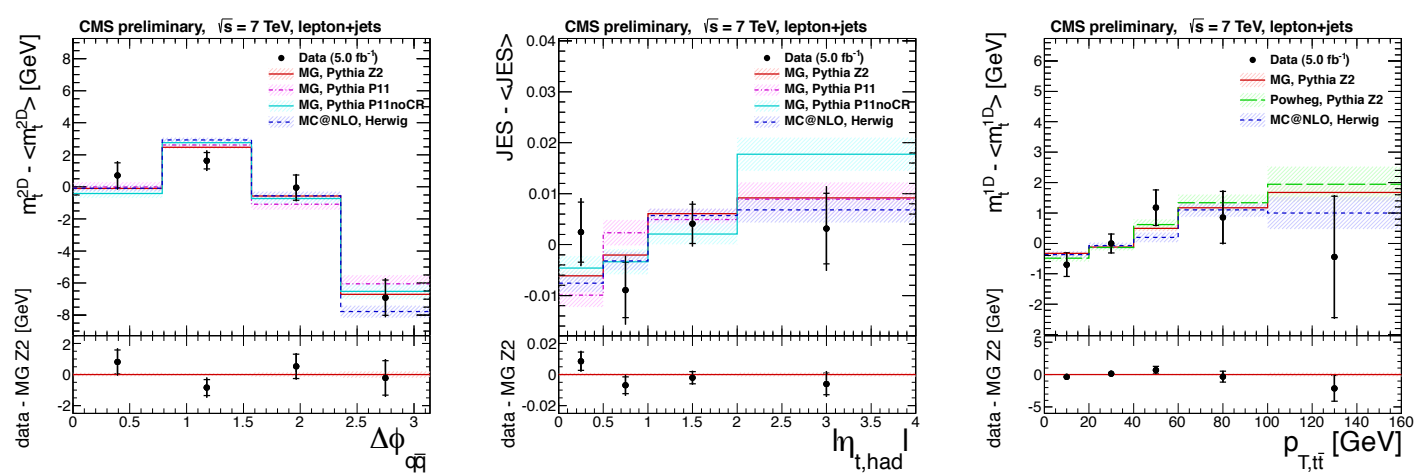

Figure 1: Two-dimensional top-quark mass measured in bins of the azimuthal opening angle between the two jets from W-boson decay (left), jet energy scale measured in bins of the pseudorapidity of the hadronically decaying top quark (middle), and one-dimensional top-quark mass measured in bins of the transverse momentum of the $t \bar{t}$ system (right).

data and the standard simulation based on MadGraph+Pythia is observed and no large effects due to colour reconnection and underlying event are seen. 


\section{The mass difference between top and antitop quarks}

One of the fundamental symmetries in the standard model, the invariance under CPT transformations, can be tested with a similar technique. The Ideogram method is used to measure the difference in mass between a particle and its corresponding antiparticle. Since the top quark decays before hadronization can take place, this difference can be measured directly. CMS performed a measurement using $19 \mathrm{fb}^{-1}$ of 2012 data in the $\ell+$ jets channel [4]. The events were splitted in two distinct samples according to the charge of the lepton. In each of these two samples, the mass of the hadronically decaying top quarks was measured and finally both masses were subtracted from eachother. This resulted in a value of $\Delta m_{t}=272 \pm 196$ (stat) \pm 122 (syst) GeV.

The smallness of the systematic uncertainty when compared to top-quark mass measurements can be explained by the cancellation of most systematic effects when taking the difference between top and antitop. The systematic uncertainty is dominated by two sources: the uncertainty on the background composition and the uncertainty on the $b$ versus $\bar{b}$ jet energy response.

\section{Extraction of the top-quark mass and $\alpha_{s}$ from the top-quark pair cross section}

One of the issues related to a top-quark mass measurement based on the invariant mass of the top decay products is the calibration on the mass used in Monte Carlo generators, which is not related in a straightforward way to the top-quark mass in a well-defined renormalization scheme like the pole mass or the $\bar{M} S$ mass. These masses can however be extracted from the measured $t \bar{t}$ production cross section, since this cross section depends on the mass of the top quark. The same procedure can also be used to extract the strong coupling constant $\alpha_{s}$ from the cross section.

The $t \bar{t}$ production cross section measured by CMS at $\sqrt{s}=7 \mathrm{TeV}$ in the dilepton channel is used together with the latest NNLO theoretical calculation of this cross section [5]. Hereby values of $m_{t}^{\text {pole }}=176.7_{-3.4}^{+3.8} \mathrm{GeV}$ and $\alpha_{s}=0.1151_{-0.0032}^{+0.0033}$ are obtained when using NNPDF. An overview of the results obtained with other PDF sets is shown in Figure 2.
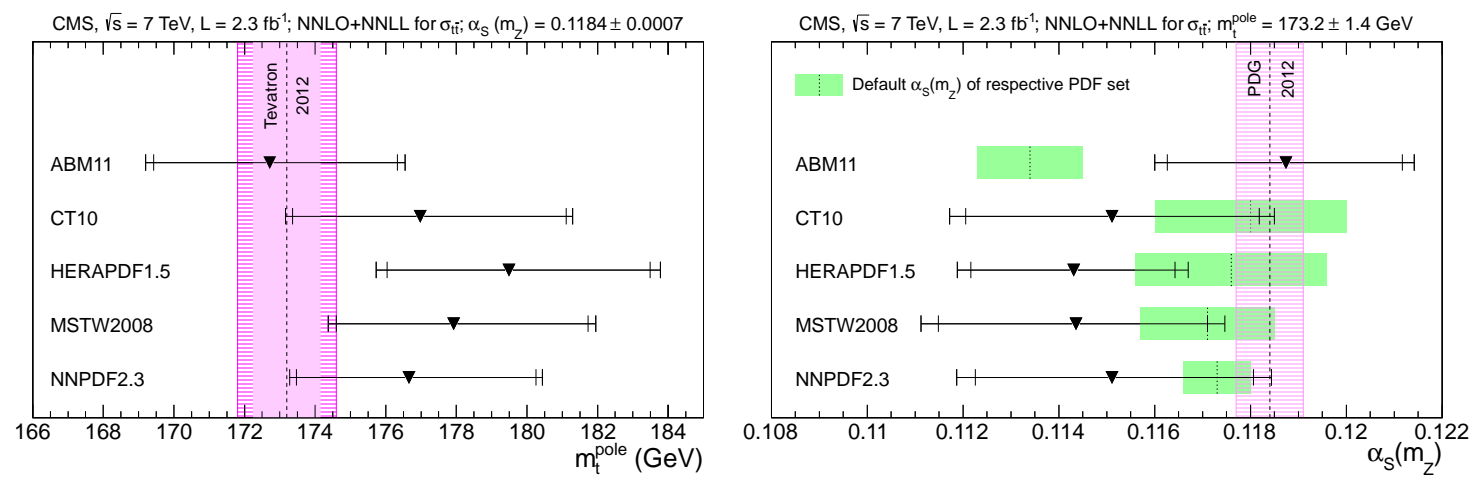

Figure 2: Values of the pole mass of the top quark (left) and the strong coupling constant $\alpha_{s}$ (right) extracted from the measured $t \bar{t}$ production cross section. 


\section{Measuring the top-quark mass via kinematic endpoints}

As previously mentioned, the standard top mass measurements are explicitly calibrated to measure the top-quark mass as defined in event generators. To overcome this problem measurements which are completely independent of simulation have to be performed, like the measurement of the top-quark mass via kinematic endpoints [6].

This analysis is based on $t \bar{t}$ events decaying in the dilepton channel. In these events the kinematic endpoints of $M_{T 2}$-like variables can be used in a fully data-driven way to simultaneously measure the mass of the top quark, the $\mathrm{W}$ boson and the neutrino or to constrain the mass of the $\mathrm{W}$ boson and the neutrino to their known values and to measure the top-quark mass. The endpoint fits for this doubly-constrained measurement are shown in Figure 3, and resulted in a value of $m_{t}=173.9 \pm 0.9$ (stat) ${ }_{-2.0}^{+1.6}$ (syst) $\mathrm{GeV}$. The systematic uncertainty on this measured value is dominated by the jet energy scale uncertainty.
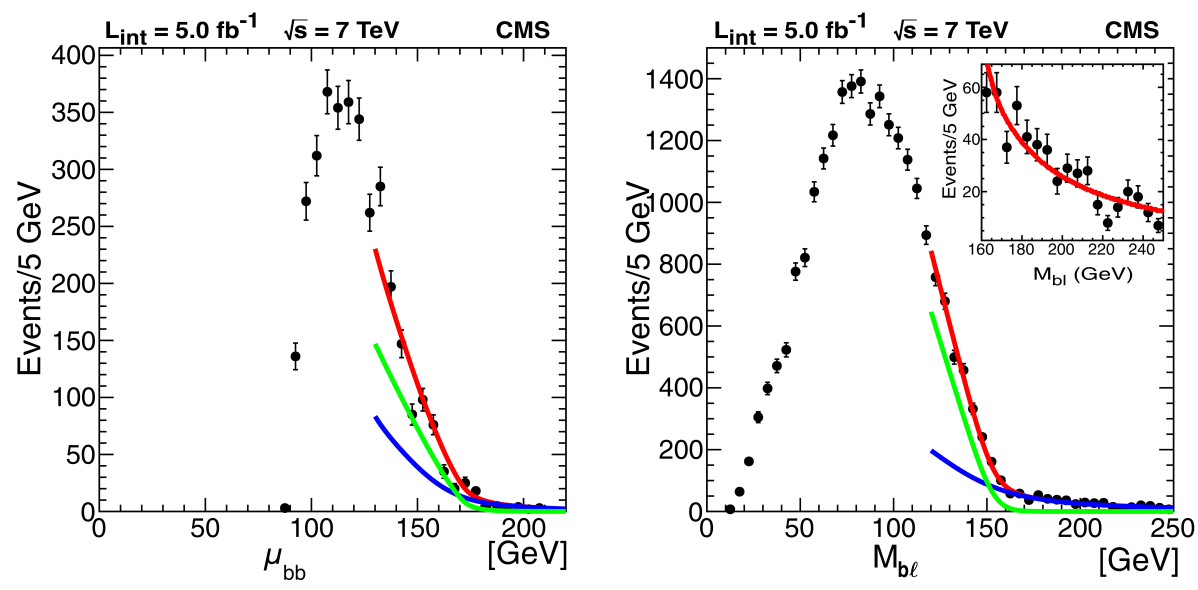

Figure 3: Endpoint fits for the doubly-constrained top-quark mass measurement.

\section{The $b$-hadron lifetime technique}

The precision on the measured top-quark mass can also be improved via alternative measurement techniques which have a different sensitivity to systematic uncertainties. A combination with the standard measurements can then result in a reduced total uncertainty. Since the decay length of the $b$-hadron from top-quark decay is correlated with the top-quark mass via the boost of the $b$ quark, the transverse decay length $L_{x y}$ of the secondary vertex is used to perform a top-quark mass measurement [7].

The dependence of the median $L_{x y}$ in the $e+$ jets, $\mu+$ jets and $e \mu$ samples on the top-quark mass is shown in Figure 4, together with the individual top-quark mass results in each channel and the combined value. The total uncertainty in every channel is dominated by the uncertainty on the modelling of the transverse momentum of the top quarks in simulation. The sub-leading systematic uncertainty in the $\ell+$ jets channels is the uncertainty on the $W+$ jets normalisation, while in the $e \mu$ channel the sub-leading systematic uncertainty is the hadronisation model uncertainty. 
CMS Simulation, $\sqrt{\mathrm{S}}=8 \mathrm{TeV}$

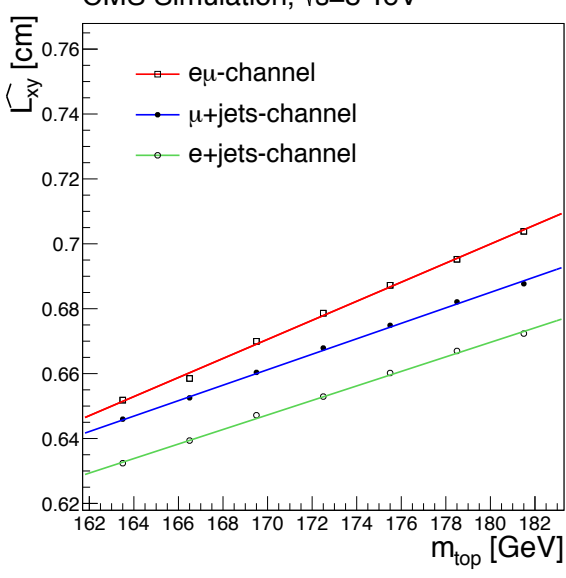

\begin{tabular}{|c|c|}
\hline Channel & $m_{t}(\mathrm{GeV})$ \\
\hline$e+$ jets & $172.8 \pm 1.0($ stat $) \pm 1.7($ syst $) \pm 3.1\left(p_{T}^{t}\right)$ \\
\hline$\mu+$ jets & $172.2 \pm 1.0($ stat $) \pm 1.6($ syst $) \pm 3.3\left(p_{T}^{t}\right)$ \\
\hline$e \mu$ & $173.7 \pm 2.0($ stat $) \pm 1.4($ syst $) \pm 2.4\left(p_{T}^{t}\right)$ \\
\hline \hline Combined & $173.5 \pm 1.5($ stat $) \pm 1.3($ syst $) \pm 2.6\left(p_{T}^{t}\right)$ \\
\hline
\end{tabular}

Figure 4: Dependence of the median transverse decay length of the secondary vertex on the top quark mass (left) and the corresponding top-quark mass measurements (right), including the combination of the three decay channels.

\section{Conclusion}

To improve our understanding of the current top-quark mass measurements and to further reduce the total uncertainty on the top-quark mass several new techniques are being explored. This includes the measurement of the kinematic dependence of the top-quark mass estimator and of the mass difference between the top and the antitop quark, the extraction of the pole mass from the $t \bar{t}$ production cross section, the usage of kinematic endpoints to extract the top-quark mass in a datadriven way, and the measurement of the top-quark mass by using the lifetime of the $b$-hadron. All these different analyses show good agreement with previous measurements and also show that the current set of simulations is able to give a proper description of top-quark mass related quantities.

\section{References}

[1] CMS Collaboration, "The CMS experiment at the CERN LHC", JINST 03 (2008) S08004.

[2] CMS Collaboration, "Measurement of the top-quark mass in t t-bar events with lepton+jets final states in pp collisions at sqrt(s) = $7 \mathrm{TeV} "$, JHEP 12 (2012) 105. arXiv: 1209.2319

[3] CMS Collaboration, "Study of the dependence of the top-quark mass measurement on event kinematics", CMS Physics Analysis Summary CMS-PAS-TOP-12-029 (2013).

[4] CMS Collaboration, "Measurement of the top - antitop mass difference in pp collisions at sqrt(s) $=8$ TeV", CMS Physics Analysis Summary CMS-PAS-TOP-12-031 (2013).

[5] CMS Collaboration, "Determination of the top-quark pole mass and strong coupling constant from the ttbar production cross section in pp collisions at sqrt(s) $=7$ TeV", Submitted to Phys. Lett. B (2013). arXiv:1307.1907

[6] CMS Collaboration, "Measurement of masses in the $\mathrm{t} t$-bar system by kinematic endpoints in $\mathrm{pp}$ collisions at sqrt(s) = 7 TeV", Eur. Phys. J. C 73 (2013) 2494. arXiv: 1304.5783

[7] CMS Collaboration, "Measurement of the top quark mass using the B-hadron lifetime technique", CMS Physics Analysis Summary CMS-PAS-TOP-12-030 (2013). 